\title{
IMPROVING BPH SYMPTOMS AND SEXUAL DYSFUNCTIONS WITH A SAW PALMETTO PREPARATION? RESULTS FROM A PILOT TRIAL
}

\section{(C) Suter A. ${ }^{1,4}$, Saller R. ${ }^{2}$, Riedi E. ${ }^{3}$, Heinrich M. ${ }^{1}$}

${ }^{1}$ The School of Pharmacy, University of London/University College of London, London, United Kingdom 'Zurich University Hospital, Institute of Natural Medicine, Zurich, Switzerland

${ }^{3}$ Urological practice, Chur, Switzerland

${ }^{4}$ A. Vogel Bioforce AG, Roggwil, Switzerland

Benign prostatic hyperplasia (BPH) is one of the main factors to cause sexual dysfunctions in elderly men. We investigated if the saw palmetto (Serenoa repens) preparation Prostasan ${ }^{\circledast}$ is efficacious and safe in treatment of patients with (BPH) and concomitant sexual dysfunctions. Eighty-two patients participated in the 8-week trial, taking one capsule of $320 \mathrm{mg}$ saw palmetto extract daily. At begin and end of the treatment period BPH symptoms and sexual dysfunctions were assessed. At the end of the treatment, $\mathrm{BPH}$ symptoms measured with the International Prostate Symptom Score was reduced from $14.4 \pm 4.7$ to
$6.9 \pm 5.2(P<0.0001)$. Sexual dysfunctions assessed with the brief Sexual Function Inventory improved from $22.4 \pm 7.2$ to $31.4 \pm 9.2(P<0.0001)$, and the Urolife BPH QoL-9 total improved from $162.7 \pm 47.9$ to $105.0 \pm 56.3(P<0.0001)$. Investigators' and patients' assessments confirmed the good efficacy, and treatment was very well tolerated and accepted by the patients. Correlation analyses confirmed the relationship between improved BPH symptoms and reduced SDys. This was the first trial with saw palmetto to show improvement in BPH symptoms and SDys as well.

\section{RANDOMIZED, DOUBLE BLIND, PLACEBO-CONTROLLED STUDY ON LONG-TERM PROPHYLAXIS WITH ECHINACEA PURPUREA}

\section{(C) Jawad M. ${ }^{1}$, Schoop R. ${ }^{2}$, Suter A. ${ }^{2}$, Eccles R.}

${ }^{1}$ Common Cold Centre and Healthcare, Cardiff University, United Kingdom

${ }^{2}$ A. Vogel Bioforce, Grünaustrasse, 9325 Roggwil(TG), Switzerland

To investigate safety and efficacy of Echinacea purpurea extract (Echinaforce ${ }^{\circledR}$ ) to prevent from cold episodes in a large population over a 4-month period. 755 healthy subjects were allocated to receive either an alcohol extract from freshly harvested E. purpurea ( $95 \%$ herba and $5 \%$ root) or placebo. Participants were required to record adverse events and to rate cold-related issues in a diary throughout the investigation period. Nasal secretions were sampled at acute colds and screened for causative particles. A total of 293 adverse events occurred with Echinacea and 306 with placebo. Nine (9) and $10 \%$ of the participants experienced adverse events, which were at least possibly related to the study drug (adverse drug reactions). With respect to safety Echinacea was non-inferior to placebo treatment.

Echinacea reduced the total number of cold episodes, cumulated episode days, and pain-killer medicated episodes significantly. Virally confirmed colds were inhibited and membranous virus infections were prevented by $53 \%(p<0.05)$. Echinacea showed effects on recurrent colds, in susceptible and immunologically vulnerable subjects. Finally, therapeutic effects increased with therapy compliance. Compliant prophylactic intake of $E$. purpurea over a 4-month period appeared to be safe and effective to prevent cold episodes. 